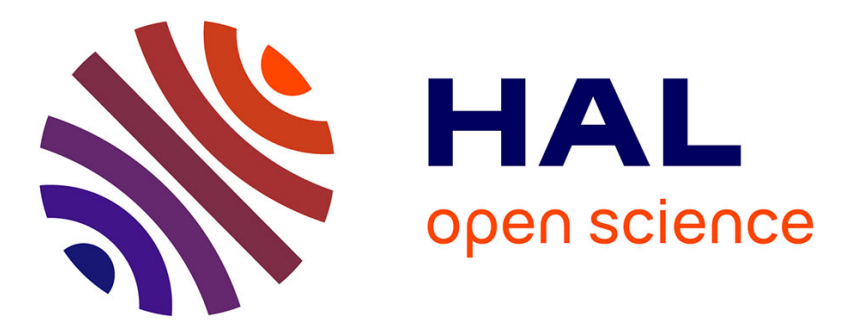

\title{
Multimodal Evaluation of the Spatio-Temporal Variations of Periprosthetic Bone Properties
}

Manon Fraulob, Sophie Le Cann, Benjamin Voumard, Hirokazu Yasui, Keita Yano, Romain Vayron, Mami Matsukawa, Philippe Zysset, Guillaume Haiat

\section{- To cite this version:}

Manon Fraulob, Sophie Le Cann, Benjamin Voumard, Hirokazu Yasui, Keita Yano, et al.. Multimodal Evaluation of the Spatio-Temporal Variations of Periprosthetic Bone Properties. Journal of Biomechanical Engineering, 2020, 142 (12), pp.121014-121023. 10.1115/1.4048399 . hal-02955178

\author{
HAL Id: hal-02955178 \\ https://hal.science/hal-02955178
}

Submitted on 1 Oct 2020

HAL is a multi-disciplinary open access archive for the deposit and dissemination of scientific research documents, whether they are published or not. The documents may come from teaching and research institutions in France or abroad, or from public or private research centers.
L'archive ouverte pluridisciplinaire $\mathbf{H A L}$, est destinée au dépôt et à la diffusion de documents scientifiques de niveau recherche, publiés ou non, émanant des établissements d'enseignement et de recherche français ou étrangers, des laboratoires publics ou privés. 


\section{Multimodal evaluation of the spatio-temporal variations of periprosthetic bone properties}

Manon Fraulob ${ }^{1}$, Sophie Le Cann ${ }^{1}$, Benjamin Voumard ${ }^{2}$, Hirokazu Yasui ${ }^{3}$, Keita Yano ${ }^{3}$, Romain Vayron ${ }^{4}$, Mami Matsukawa ${ }^{3}$, Philippe Zysset ${ }^{2}$, Guillaume Haïat ${ }^{1 *}$

${ }^{1}$ CNRS, Univ Paris Est Creteil, MSME UMR 8208, F-94010 Creteil, France

${ }^{2}$ ARTORG Center for biomedical engineering research, University of Bern, Bern, Switzerland

${ }^{3}$ Laboratory of Ultrasonic Electronics, Applied Ultrasonic Research Center, Doshisha University, Kyotanabe, 610-0321 Kyoto, Japan

${ }^{4}$ Université Polytechnique Hauts de France, Laboratoire d'Automatique, de Mécanique et d'informatique Industrielles et Humaines, LAMIH UMR CNRS 8201, Valenciennes, France

\section{* CORRESPONDING AUTHOR:}

Guillaume Haiat

Laboratoire Modélisation et Simulation Multi Echelle (MSME), Equipe Biomécanique Université Paris-Est Créteil Val de Marne (UPEC), Faculté des Sciences et Technologie 61, Avenue du Général de Gaulle 94010 Créteil Cedex - FRANCE

E-mail: Guillaume.HAIAT@cnrs.fr 


\section{Abstract.}

Titanium implants are widely used in dental and orthopedic surgery. However, implant failures still occur because of a lack of implant stability. The biomechanical properties of bone tissue located around the implant need to be assessed to better understand the osseointegration phenomena and anticipate implant failure. The aim of this study was to explore the spatio-temporal variation of the microscopic elastic properties of newly formed bone tissue close to an implant.

Eight coin-shaped Ti6AI4V implants were inserted into rabbit tibiae for 7 and 13 weeks using an in vivo model allowing the distinction between mature and newly formed bone in a standardized configuration. Nanoindentation and micro-Brillouin scattering measurements were carried out in similar locations to measure the indentation modulus and the wave velocity, from which relative variations of bone mass density were extracted.

The indentation modulus, the wave velocity and mass density were found to be higher i) in newly formed bone tissue located close to the implant surface, compared to mature cortical bone tissue, and ii) after longer healing time, consistently with an increased mineralization. Within the bone chamber, the spatial distribution of elastic properties was more heterogeneous for shorter healing durations. After 7 weeks of healing, bone tissue in the bone chamber close to the implant surface was $12.3 \%$ denser than bone tissue further away. Bone tissue close to the chamber edge was $16.8 \%$ denser than in its center. These results suggest a bone spreading pathway along tissue maturation, which is confirmed by histology and consistent with contact osteogenesis phenomena.

KEYWORDS: bone, implant, nanoindentation, micro-Brillouin scattering, osseointegration 


\section{Introduction}

Titanium implants are widely used in dental, maxillo-facial and orthopedic surgery. However, surgical failures still occur, inducing pain, additional surgery and important additional costs for the healthcare system. Implant failures remain difficult to anticipate and are often related to aseptic loosening, which corresponds to a lack of implant stability [1].

Two kinds of implant stability may be distinguished and are strong determinants of the surgical success. The primary implant stability is obtained immediately after surgery and depends mostly on the surgical procedure, the implant design and local bone properties at the implantation site [2]. A few weeks or months after surgery, primary stability is replaced by secondary implant stability when bone tissue heals and forms around the implant, which corresponds to the osseointegration phenomena [2]. Newly formed bone tissue is created and grows in direct contact with the implant surface, leading to the formation of a bone-implant interface (BII) $[2,3]$. The surgical success of an implant is determined by the bone quantity and quality at the BII, especially in a region of interest up to $200 \mu \mathrm{m}$ from the implant surface $[2,4]$.

During osseointegration, periprosthetic bone properties evolve due to formation, maturation and remodeling of bone tissue in order to adapt to the mechanical environment [5], which leads to intertwined changes of bone quantity, composition and structure, ultimately defining the tissue mechanical properties. While bone quantity and microstructure can be retrieved from histological analysis [4] and micro-computed tomography [5], bone quality is more difficult to assess since it depends on bone composition and nanostructure, that can be measured for example by Raman spectroscopy [6] and diffraction techniques [7]. The resulting bone mechanical properties can be assessed at different length scales, e.g. microscopically with nanoindentation $[8,9]$ or globally through implant pullout [10].

Due to their specific geometry, the use of clinical implants leads to complex and multiaxial stress distribution at the BII, which may affect the evolution of the biomechanical properties of the BII. Moreover, in clinical models, it remains difficult to clearly distinguish between mature and newly formed bone tissues. To overcome such limitations, a coin-shaped implant model with a planar interface has been developed [11-15] to be inserted into cortical bone. This animal model allows a standardized geometry and a controlled stress field around the BII. More recently, a $200 \mu \mathrm{m}$-thick gap (referred to as "bone chamber") has been considered between the implant surface and bone tissue [4, 16]. During healing, this bone chamber is filled with blood and soft tissue and later with newly formed bone tissue, thus clearly distinguishable from pre-existent mature tissue. This animal model has proven its effectiveness to allow the characterization of bone properties using various techniques such as 
quantitative ultrasound [17], mechanical cleavage experiments [18] or nanoindentation and microBrillouin analyses as detailed hereafter.

Nanoindentation has been widely employed to investigate the local bone biomechanical properties at the microscopic scale, such as the evolution of the elastic modulus with age [19-21], with the risk of fracture [22] and during bone healing [23, 24]. Nanoindentation has been also used to measure the elastic properties of engineered bone obtained by transplantation of scaffolds seeded with mesenchymal stem cells $[25,26]$, and to evaluate the effect of bone tissue mineral content [27], nanostructure [28] and anisotropy on the microscopic elastic properties, both experimentally and numerically $[29,30]$. The elastic properties of bone tissue at the Bll have also been characterized around dental implants to assess the effect of implant material and surface treatment [31-33], of location around the implant [8,32], of mechanical loading [34] and of bone maturation and healing $[33,35]$. In another in vivo model where a titanium plate has been subperiosteally implanted into cortical bone, nanoindentation has been used to characterize the differences of elastic properties between bone tissue developing at the implant surface and mature bone [36]. Nanoindentation has been conducted using the aforementioned in vivo standardized implant model to compare the properties of mature and newly formed bone tissues and evaluate their evolution during healing [37]. However, no spatial analysis of the variation of the bone properties has yet been conducted, which would be useful in order to clarify how bone tissue forms and spreads at the implant surface.

Micro-Brillouin scattering is another technique that can be used to investigate the elastic properties of materials, based on photoelastic effects. Micro-Brillouin scattering allows the assessment of the ultrasonic wave velocity of the tested material in the $\mathrm{GHz}$ range, without contact and nondestructively [38]. Micro-Brillouin scattering has been used to investigate bone wave velocity, in particular within the femoral head [39] or in a bone defect [40], as well as to assess bone anisotropic properties [41], bone structure and alignment [42], the effect of decalcification [43] and the effects of glycation [44]. At the Bll, only limited data is available. To the best of the authors' knowledge, micro-Brillouin scattering has only been applied using the coin-shaped implant in vivo model, in order to compare wave velocities between peri-implant and mature bone tissue $[16,45]$, and no evaluation of the spatial distribution of wave velocity has yet been carried out.

Multimodal analysis has proven its effectiveness to characterize multiple bone properties in sitematched locations at the BII [8, 45-47]. The combination of nanoindentation and micro-Brillouin scattering may be used to investigate complementary elastic properties at the microscale. Nanoindentation quantifies bone tissue elastic modulus while micro-Brillouin scattering measures the ultrasonic wave velocity. Considering bone tissue as an isotropic material, bone mass density can be 
expressed as the ratio of the Young's modulus and of the square of the ultrasonic velocity, which represents a powerful approach to retrieve mass density variations at the scale of several micrometers [45]. In a previous study [45], both techniques were combined to show that after 7 weeks of healing time, mature bone was $12.2 \%$ denser than newly formed bone, which was explained by a lower degree of mineralization of newly formed bone tissue. However, the spatial differences within the bone chamber were not investigated. Yet, assessing the spatio-temporal variation of the biomechanical properties of periprosthetic bone tissue is of interest to better understand the osseointegration process, in a long-term clinical goal to optimize implant stability and surgical success [48].

The aim of this study is to investigate the evolution of the biomechanical properties of periprosthetic newly formed bone tissue as a function of healing time and of location relatively to the implant surface. To do so, a standardized bone chamber model enables the characterization of newly formed bone by isolating it from pre-existing mature cortical bone. Using this implant model, nanoindentation, histological analysis and micro-Brillouin scattering are combined at site-matched locations in a multimodal experiment, to retrieve spatio-temporal variation of multiple properties.

\section{Methods}

\subsection{Implants}

Eight coin-shaped implants ( $5 \mathrm{~mm}$ diameter, $3 \mathrm{~mm}$ length) made of medical grade titanium alloy, Ti6Al4V, were produced. The surface in contact with bone was mirror polished and then sandblasted with aluminium oxide $\left(\mathrm{Al}_{2} \mathrm{O}_{3}\right)$ powder of grain size of $250 \mu \mathrm{m}$ (Cobra, Renfert, Hilzingen, Germany) for 30 seconds at 6 bars using the Basic Quattro device (Renfert, Hilzingen, Germany). This protocol led to a surface roughness $S_{a}=3.46 \pm 0.25 \mu \mathrm{m}$ as measured by topographical analysis (Alicona Infinite Focus, Raaba, Austria), chosen to be close to the range of values [3.6 - 3.9 $\mu \mathrm{m}$ ] leading to an optimal osseointegration [11].

The implants were surrounded by a polytetrafluoroethylene (PTFE) cap to avoid bone attachment on the lateral sides and to create a $200 \mu \mathrm{m}$-thick chamber within the cortical bone region underneath the implant to be filled by newly formed bone tissue after surgery (see Fig. 1).

Before being autoclaved $\left(1.5 \mathrm{~atm}\right.$ at $121^{\circ} \mathrm{C}$ for $20 \mathrm{~min}$ ), all implants were rinsed with ethanol and placed in an ultrasonic bath with absolute ethanol $(20 \mathrm{~min}$ ) and then with demineralized water (30 $\mathrm{min})$. 

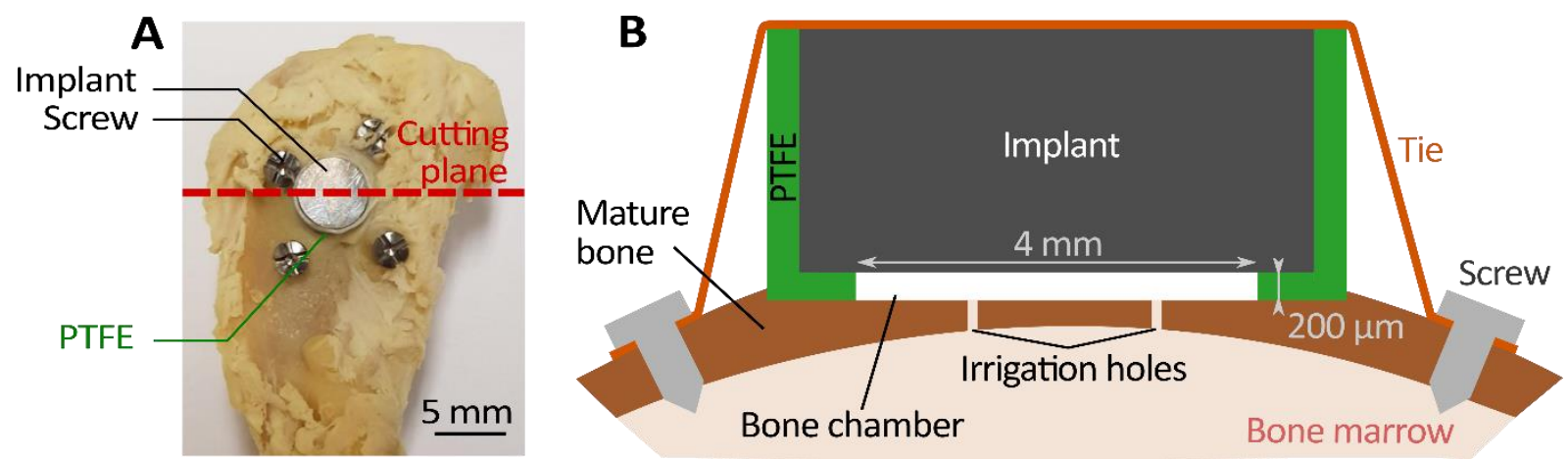

Figure 1 : A) Coin-shaped implant inserted into the proximal-medial part of a rabbit tibia, B) Schematic crosssection of the coin-shaped implant model

\subsection{Surgical procedure}

The implants were inserted into the proximal-medial part of the right tibia of eight New Zealand white male rabbits (average weight $3.9 \mathrm{~kg}$ ), as shown in Fig. 1A. As in [16], the animals were anesthetized via intramuscular injection of $0.5 \mathrm{mg} / \mathrm{kg}$ Diazepan (Valium ${ }^{\circledR}$; Roche, Basel, Switzerland), $0.25 \mathrm{mg} / \mathrm{kg}$ metedomidine hydrochloride (Domitor; Virbac, Carros, France) and $100 \mathrm{mg} / \mathrm{kg}$ ketamine hydrochloride (Ketalar500 ${ }^{\circledR}$; Pfizer, New York, NY, USA). The implantation site was exposed and a $5.6 \mathrm{~mm}$-diameter cavity and four $1.2 \mathrm{~mm}$-diameter irrigation holes were drilled with a surgical system (Zimmer, Palm Beach Gardens, FL, USA) in order to create a planar cortical bone surface to receive the implant and to allow fluid flow from the medullary canal towards the interface. Four other $1.2 \mathrm{~mm}$-diameter holes were drilled around the implant to receive $1.6 \mathrm{~mm}$-diameter osteosynthesis screws (Easy Implant, Chavanod, France) to maintain the implant, linked by two elastic strings in a cross-pattern (Fig. 1).

After surgery, $25 \mu \mathrm{g} / \mathrm{h}$ fentanyl were transdermally delivered for 3 days and $1 \mathrm{~g} / 100 \mathrm{ml}$ enrofloxacine was put in water for 5 days. The animals were housed in a metal hutch in an environment (ambient temperature $19{ }^{\circ} \mathrm{C}$ and humidity of $55 \%$ ) in accordance with the requirements of the European Guidelines for care and use of laboratory animals and the ethical committee of ENVA (Ecole Nationale Vétérinaire d'Alfort). Artificial lightening and air conditioning systems were used in the animal housing facility. The animals were fed with commercial food and water was provided ad libitum.

Four rabbits were euthanized with an overdose of pentobarbital after a healing time of 7 weeks and four other rabbits were euthanized after a healing time of 13 weeks.

\subsection{Sample preparation}

After sacrifice, all samples were embedded in polymethyl methacrylate (PMMA) in order to cut the BII without damaging it. The embedding protocol consisted in fixing the tissues for one week in $10 \%$ 
phosphate-buffered formalin, rinsing with water, dehydrating in ethanol and clearing in xylene before embedding it into methyl methacrylate (MMA) $[49,50]$.

The embedded samples could then be cut in the transverse plane (see Fig. 1A) using a low-speed cutoff machine (Minitom, Struers ${ }^{\circledR}$, Ballerup, Danemark). Two slices were produced for each sample. The first slice was $800 \mu \mathrm{m}$-thick and was cut approximately in the middle of the sample. It was glued on a Permanox slice (Thermo Scientific Nunc, Waltham, USA) and its surface was polished (PM5, Logitech ${ }^{\circledR}$, Glasgow, UK) with abrasive paper, SiC Foil with grain size of 1200 and 2000 grit and polishing cloths with $0.3-\mu \mathrm{m}$ aluminium oxide powder until the sample reached a thickness of $500 \mu \mathrm{m}$. The slice was finally rinsed in an ultrasonic bath with $0.9 \% \mathrm{NaCl}$ for $20 \mathrm{~min}$. The prepared slice was first analyzed by nanoindentation (see subsection 2.5), before being stained for histological analysis (see subsection 2.4).

The second slice was $400 \mu \mathrm{m}$-thick and both sides were manually polished (Minitom, Struers ${ }^{\circledR}$, Ballerup, Denmark) using abrasive paper, SiC Foil with grain size of 1200 grit until the thickness was reduced to approximately $200 \mu \mathrm{m}$ thanks to a sample holder controlling the removal of material (AccuStop, Struers ${ }^{\circledR}$, Ballerup, Denmark). This thinnest slice was analyzed by micro-Brillouin scattering (see subsection 2.6).

\subsection{Histological analysis}

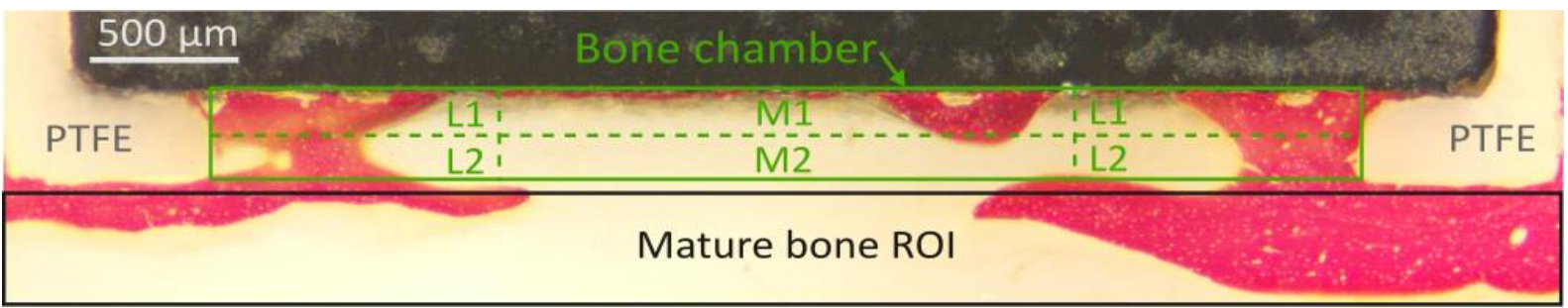

Figure 2 : Microscopic image obtained after van Gieson picro-fuchsin staining of a $500 \mu \mathrm{m}$-slice after 13 weeks of healing time. Bone tissue is colored in red and the implant in black. The yellow regions of interest correspond to soft non-mineralized tissue. The green rectangular region of interest (ROI) corresponds to the bone chamber partly colonized with newly formed bone, which is divided into 4 subregions of interest: lateral $(\mathrm{L} 1, \mathrm{~L} 2)$ and central $(\mathrm{M} 1, \mathrm{M} 2)$. The black rectangular ROI corresponds to the region where measurements were taken in mature cortical bone.

For the histological analyses, the slices were stained with van Gieson picro-fuchsin for 1 min and then rinsed in absolute ethanol. After rinsing, the dye fixed on collagen fibers colored bone tissues in red, as shown in Fig. 2. The regions which were not colored in red correspond to non-mineralized soft tissues (such as bone marrow). Four regions of interest (ROIs) were defined within the bone chamber 
partially filled with newly formed bone without any mature bone: two lateral ROIs (L1 and L2) and two central ROIs (M1 and M2).

Each $500 \mu$ m-thick slice was imaged by light microscopy with Stemi 305 (Zeiss ${ }^{\circledR}$, Jena, Germany). For each specimen, the bone chamber was isolated and bone tissue was visually segmented using ImageJ ( $\mathrm{NIH}$, Bethesda, USA) [51]. For all 4 ROIs (see Fig. 2), the ratio between the bone area (i.e. segmented bone pixels) and the total area (i.e. all pixels in the ROI) $(B A / T A)$ was calculated using the BoneJ plugin [52].

\subsection{Nanoindentation measurements}

Nanoindentation analyses were carried out along the bone axis with a nanoindenter (UNHT, CSM Instrument, Switzerland) equipped with a Berkovich diamond indentation tip under dry conditions. The indentation depth reached a maximum value of $1 \mu \mathrm{m}$ during a $30 \mathrm{~s}$ holding time after a loading phase (loading rate: $100 \mathrm{mN} / \mathrm{min}$ ) and before an unloading phase (unloading rate: $400 \mathrm{mN} / \mathrm{min}$ ).

Prior to all measurements, the device was calibrated by performing measurements in fused silica. In each lateral (L1 and L2) and central (M1 and M2) ROI, nanoindentation measurements were carried out in sixteen locations that were manually selected. In mature bone tissue, forty measurements were carried out below the chamber, to collect relevant control values obtained in mature cortical bone with corresponding anatomical location, at a distance from the bone chamber comprised between 40 $\mu \mathrm{m}$ and $400 \mu \mathrm{m}$ (black rectangular ROI in Fig. 2). All locations were chosen in order to avoid indenting PMMA and were separated from each other (respectively from the implant surface) by a minimum distance of $14 \mu \mathrm{m}$ (respectively $7 \mu \mathrm{m}$ ).

For each measurement, the variation of the force as a function of the penetration depth was retrieved and the contact point identified. The reduced modulus $E_{r}$ was determined [53] from the maximal slope of the load-penetration curve at the beginning of the unloading phase assuming bone tissue as a homogeneous, linear elastic, and isotropic material. The indentation modulus $E^{*}$ was determined following Eq. (1) considering a Young's modulus $E_{i}=1141 \mathrm{GPa}$ and a Poisson's ratio $v_{i}=0.07$ for the diamond indenter tip:

$$
\frac{1}{E_{r}}=\frac{1}{E^{*}}+\frac{1-v_{i}{ }^{2}}{E_{i}},
$$

\subsection{Micro-Brillouin scattering measurements}

Micro-Brillouin scattering measurements were conducted using a six-pass tandem Fabry-Pérot interferometer (JRS Scientific Instruments, Zurich, Switzerland) using a $532 \mathrm{~nm}\left(\lambda_{0}\right)$ solid-state laser (Spectra Physics, Excelsior, Santa Clara, CA). The samples were fixed on a flat metal reflector and placed 
with a controlled angle of $\Theta / 2$ between the sample's normal and the incident laser beam (Fig. 3A). The laser spot had an approximate resolution of $10 \mu \mathrm{m}$ and a power of $12 \mathrm{~mW}$ avoiding any heat effect.
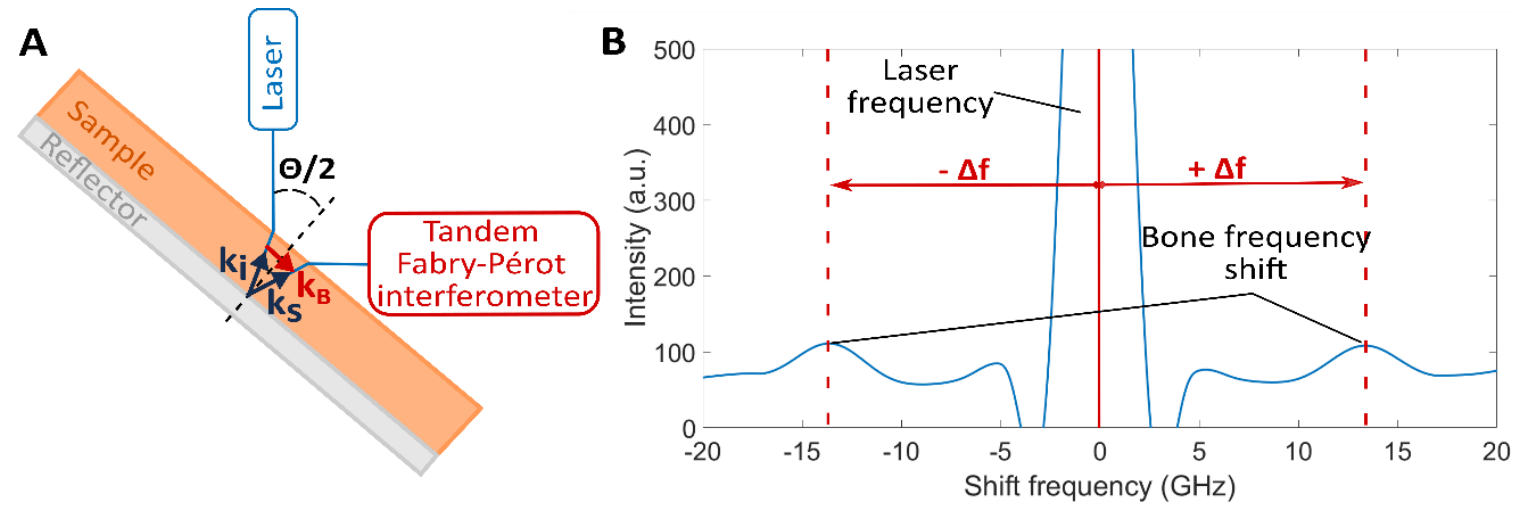

Figure 3 : A) Schematic representation of reflection induced $\Theta$ angle (RIOA) micro-Brillouin scattering measurement configuration, B) Micro-Brillouin spectrum and bone frequency shifts (denoted by $\Delta f$ ). The spectrum represents the filtered (1D median filter) and smoothed data for one given measuring point corresponding to a wave velocity equal to $4994 \mathrm{~m} / \mathrm{s}$.

The incident laser beam (vector $k_{i}$ ) propagated through the studied sample following Snell-Descartes laws (Fig. $3 \mathrm{~A}$ ) and phonons (vector $k_{B}$ ) were created because of Brillouin scattering effects arising from the interaction between light and bone tissue, leading to a frequency shift of the scattered beam (vector $k_{s}$ ). The scattered beam was detected with a photomultiplier (Hamamatsu, R464s, Shizuoka, Japan) and averaged from Sandercock Type Tandem Fabry-Pérot interferometer (The table stable, Mettmenstetten, Switzerland) scans with a photon counter. After a Voigt fitting on the obtained spectrum (Fig. 3B), the frequency shifts $\Delta f$ between the incident laser beam and the resultant scattered beam were used to access to the wave velocity in the plane of the sample surface, $v$, following Eq. (2) [54]:

$$
v=\frac{\Delta f \cdot \lambda_{0}}{2 \cdot \sin (\Theta / 2)}
$$

where $\lambda_{0}=532 \mathrm{~nm}$ is the laser wavelength and $\Theta / 2=45^{\circ}$ is the angle between the sample's normal and the incident laser beam.

An optical microscope (Photon design, Mercure, Tokyo, Japan) allowed the selection of the measurement location. One measurement point was selected within each of the newly formed bone ROIs when enough bone tissue was present, and six measurement points were considered within the mature bone ROI. For micro-Brillouin measurements, the ultrasonic waves propagate in the plane of the sample, which corresponds to the plane perpendicular to the bone axis (see Fig. 1A). For each measuring point, measurement was repeated five times leading to an average value of five velocities. The measurement errors obtained in [55] are of the order of $1 \%$. 


\subsection{Statistical analysis}

Due to the relatively low number of measurements per ROI, values were averaged among larger groups as defined in Table 1.

Table 1 : Definition of the groups used in the statistical analysis and derived from the bone chamber regions of interest defined in Fig. 2

\begin{tabular}{|l|l|}
\hline Group & Regions \\
\hline Newly formed bone & $\mathrm{L} 1+\mathrm{L} 2+\mathrm{M} 1+\mathrm{M} 2$ \\
\hline Close & $\mathrm{L} 1+\mathrm{M} 1$ \\
\hline Far & $\mathrm{L} 2+\mathrm{M} 2$ \\
\hline Lateral & $\mathrm{L} 1+\mathrm{L} 2$ \\
\hline Central & $\mathrm{M} 1+\mathrm{M} 2$ \\
\hline
\end{tabular}

For all groups mentioned in Table 1 and for mature bone, the average and standard deviation values of $E^{*}$ (nanoindentation), $v$ (micro-Brillouin scattering) and BA/TA (histology) were determined for all samples corresponding to a healing time of 7 weeks and of 13 weeks. The results were compared using non-parametric Mann-Whitney U-tests (Matlab R2017b, MathWorks ${ }^{\circledR}$, Natick, USA) to assess the statistical significance between the groups.

\subsection{Local density estimation}

Combining nanoindentation and micro-Brillouin scattering results can provide an estimation of the relative variations of bone mass density $\rho$ at the scale of several micrometers, since $\rho$ is linked to wave velocity $v$ and Young's modulus $E$ assuming an isotropic elastic constitutive law following:

$$
v=\sqrt{\frac{E}{\rho}}
$$

Please refer to the limitations paragraph at the end of section 4 for a discussion about the isotropic assumption of bone tissue.

The differentiation of Eq. (3) leads to:

$$
\frac{\Delta \rho}{\rho_{0}}=\frac{\Delta E}{E_{0}}-2 \frac{\Delta \mathrm{v}}{v_{0}^{\prime}}
$$

where $\Delta E, \Delta v$ and $\Delta \rho$ (respectively, $E_{0}, v_{0}$ and $\rho_{0}$ ) represent the differences between the values (respectively, the average values) of $E, v$ and $\rho$ for two studied groups. 
Note that the relationship between the indentation modulus $E^{*}$ and the Young's modulus $E$ is given by:

$$
E=E^{*}\left(1-v_{b}^{2}\right)
$$

where the Poisson's ratio of bone tissue $v_{b}=0.3$ was chosen based on $[8,31,35]$.

\section{Results}

\subsection{Histological analysis}

Figure 4 shows the difference of the distribution of the values of $B A / T A$ corresponding to samples obtained after 7 and 13 weeks of healing time within the bone chamber. At 13 weeks of healing time, the bone ratio $B A / T A$ is significantly lower for the far group compared to the close group (Fig. 4A) as well as for the central group compared to the lateral group (Fig. 4B). However, even if the same trend is obtained for samples corresponding to 7 weeks of healing time, the differences are not significant. For all the groups, the value of the $B A / T A$ is non-significantly higher after 13 weeks of healing time compared to 7 weeks. Moreover, the value of the standard deviations decreases as a function of healing time for all groups.
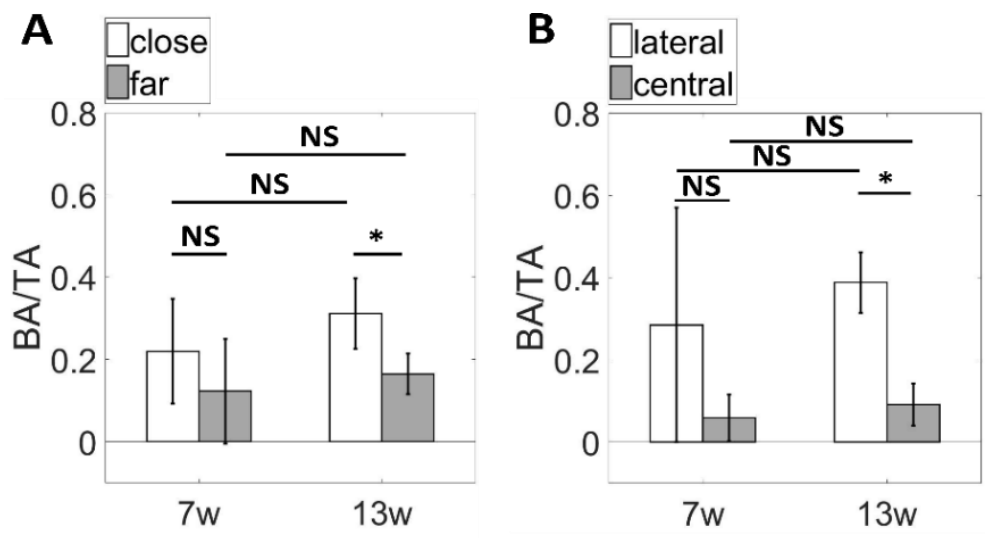

Figure 4 : Distribution (average and standard deviation values) of bone ratios (BA/TA) after 7 weeks (7w) and 13 weeks $(13 w)$ of healing time for A: the close and far groups and B: the lateral and central groups. The MannWhitney U-test analysis leads to: ${ }^{*}=p$-value $\leq 0.05, N S=p$-value $>0.05$ (non-significant difference).

\subsection{Nanoindentation measurements}

Figure $5 \mathrm{~A}$ shows the distribution of the indentation modulus $E^{*}$ in mature and newly formed bone tissues. At both healing times ( 7 and 13 weeks), the indentation modulus $E^{*}$ is significantly higher in mature than in newly formed bone tissue. Moreover, $E^{*}$ is significantly higher after 13 weeks of healing time compared to 7 weeks of healing time in newly formed bone tissue, whereas it does not vary significantly in mature bone tissue. 
Figure $5 B$ shows that after 7 weeks of healing time, the indentation modulus $E^{*}$ is significantly lower in the far group than in the close group, while no significant differences are observed after 13 weeks. Moreover, when considering separately the far and close groups, the values of $E^{*}$ are significantly higher after 13 weeks of healing time compared to 7 weeks of healing time.

Similar results are obtained when considering the lateral and central groups (Fig. 5C). After 7 weeks of healing time, the values of $E^{*}$ are significantly lower within the central group than within the lateral group, while no significant differences are observed after 13 weeks of healing time. Moreover, when considering the central and lateral groups individually, the values of $E^{*}$ are significantly higher after 13 weeks of healing time compared to 7 weeks of healing time.
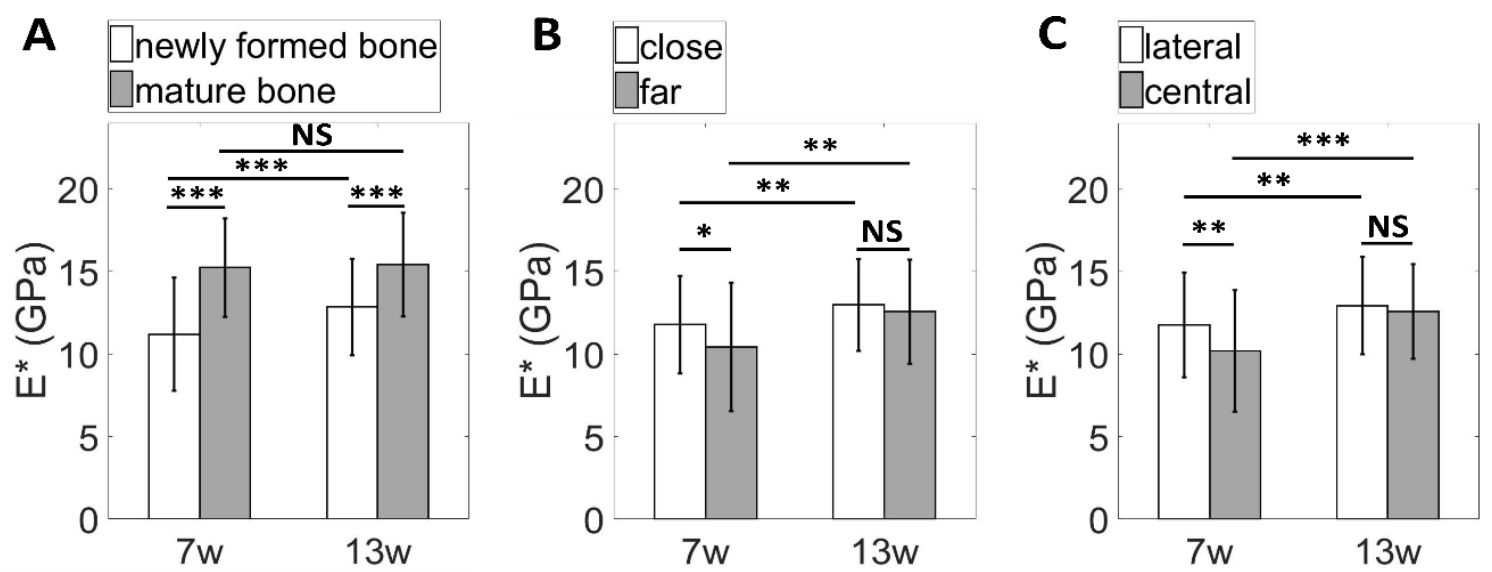

Figure 5 : Distribution (average and standard deviation values) of the indentation modulus $\mathrm{E}^{*}$ after 7 weeks $(7 w)$ and 13 weeks $(13 w)$ of healing time, based on the groups defined in Table 1. A: mature vs newly formed bone tissue, B: close vs far within the bone chamber and C: lateral vs central within the bone chamber. The Mann-Whitney U-test analysis leads to: ${ }^{*}=p$-value $\leq 0.05, * *=p$-value $\leq 0.01, * * *=p$-value $\leq 0.001, N S=p$ value $>0.05$ (non-significant difference).

\subsection{Micro-Brillouin scattering measurements}

Figure $6 \mathrm{~A}$ shows the distribution of the wave velocities $v$ in mature and newly formed bone tissues after 7 and 13 weeks of healing times. After both healing times, the wave velocity $v$ is significantly higher in mature than in newly formed bone tissue. Moreover, the wave velocities are significantly higher for both newly formed and mature bone tissues after 13 weeks of healing time compared to 7 weeks of healing time.

As shown in Fig. 6B, within the bone chamber, no significant differences in wave velocities are observed between the far and the close group after 7 or 13 weeks of healing time. However, when considering 
separately the far and close groups, the values of $v$ are significantly higher after 13 weeks of healing time compared to 7 weeks of healing time.

Similar results are obtained when considering the lateral and central groups (Fig. 6C). No significant differences are obtained between the lateral and central groups after 7 or 13 weeks of healing time. However, when considering the lateral group only, the values of $v$ are significantly higher after 13 weeks of healing time compared to 7 weeks of healing time, whereas the increase of $v$ is not significant for the central group.
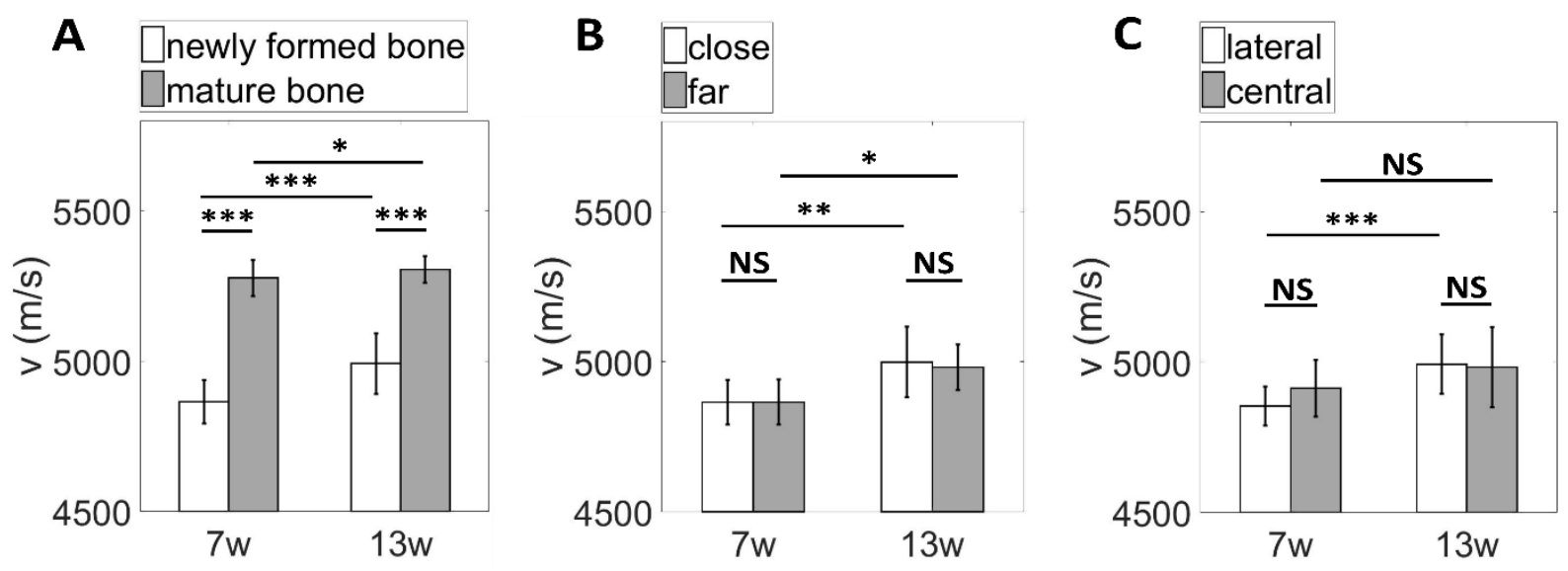

Figure 6 : Distribution (average and standard deviation values) of the wave velocity $v$ after 7 weeks (7w) and 13 weeks (13w) of healing time. A: mature vs newly formed bone tissue, B: close vs far groups and C: lateral vs central groups. The Mann-Whitney U-test analysis leads to: ${ }^{*}=\mathrm{p}$-value $\leq 0.05, * *=p$-value $\leq 0.01, * * *=p$ value $\leq 0.001, \mathrm{NS}=\mathrm{p}$-value $>0.05$ (non-significant difference) .

\subsection{Local density estimation}

Table 2 shows the relative variations of $E, v$ and $\rho$ obtained when comparing i) mature and newly formed bone tissues, ii) close and far groups and iii) central and lateral groups. The positive values indicate that all three parameters have the same general evolution increasing from i) newly formed to mature bone, ii) the far to close group and iii) the central to lateral group for both healing times ( 7 and 13 weeks). Only the variation of velocity $v$ after 7 weeks of healing has a negative value. However, for all compared groups, the variations of $v$ are smaller than those of $E$ and $\rho$. Furthermore, the variations of $E$ and $\rho$ are larger after 7 weeks than 13 weeks of healing time for all groups. 
Table 2 : Relative variations of $\mathrm{E}, \mathrm{v}$ and $\rho$ obtained when comparing i) mature and newly formed bone tissues, ii) the far and close groups and iii) the central and lateral groups after 7 and 13 weeks of healing time. The data corresponding to the relative variation of E are obtained from Fig. 5 using Eq. (5). Positive values indicate an increase of the parameters from newly formed to mature bone, from far to close groups and from central to lateral groups.

\begin{tabular}{|c|c|c|c|}
\hline Compared groups & Parameters & 7 weeks of healing & 13 weeks of healing \\
\hline $\begin{array}{c}\text { i) newly formed bone } \\
\text { vs mature bone }\end{array}$ & $\Delta \mathrm{E} / \mathrm{E}_{0}$ & $30,5 \%$ & $18,3 \%$ \\
& $\Delta \rho / \mathrm{v}_{0}$ & $8,1 \%$ & $6,1 \%$ \\
& $\Delta \mathrm{E} / \mathrm{E}_{0}$ & $14,3 \%$ & $6,1 \%$ \\
ii) far vs close & $\Delta \mathrm{v} / \mathrm{v}_{0}$ & $12,3 \%$ & $3,2 \%$ \\
& $\Delta \rho / \rho_{0}$ & $12,3 \%$ & $0,3 \%$ \\
& $\Delta \mathrm{E} / \mathrm{E}_{0}$ & $14,4 \%$ & $2,5 \%$ \\
\hline iii) central vs lateral & $\Delta \mathrm{v} / \mathrm{v}_{0}$ & $-1,2 \%$ & $2,8 \%$ \\
& $\Delta \rho / \rho_{0}$ & $16,8 \%$ & $0,2 \%$ \\
\hline
\end{tabular}

\section{Discussion}

The originality of this multiphysics experimental study is to combine for the first time nanoindentation, micro-Brillouin scattering and histology, in order to investigate the evolution of the biomechanical properties of periprosthetic bone tissue during osseointegration. By isolating newly formed bone tissue within a bone chamber divided into 4 subregions for two healing durations ( 7 and 13 weeks), the spatio-temporal variations of $2 \mathrm{D}$ bone distribution $B A / T A$, indentation modulus $E^{*}$, ultrasonic wave velocity $v$ and bone mass density $\rho$ were evaluated.

The average indentation modulus $E^{*}$ obtained herein is equal to $11.2 \mathrm{GPa}$ (respectively $12.84 \mathrm{GPa}$ ) within newly formed bone and to $15.23 \mathrm{GPa}$ (respectively $15.42 \mathrm{GPa}$ ) in mature cortical bone after 7 weeks (respectively 13 weeks) of healing time. The averaged Young's modulus $E$ (derived using Eq. (5)) 
obtained herein is equal to $10.19 \mathrm{GPa}$ (respectively $11.68 \mathrm{GPa}$ ) in newly formed bone and of $13.86 \mathrm{GPa}$ (respectively $14.03 \mathrm{GPa}$ ) in mature cortical bone after 7 weeks (respectively 13 weeks) of healing time. Here, the bone Poisson's ratio was chosen based on the literature but different values have also been considered [56]. A variation of the bone Poisson's ratio from 0.2 to 0.4 leads to a decrease of the bone Young's modulus equal to $13 \%$ and does not affect the relative variation of the bone Young's modulus obtained between the different groups in Table 2 (see Table 3). These values are consistent with literature data collected on rabbit femurs, where Young's moduli ranged from 6-12 GPa for newly formed bone tissue and of $9-17 \mathrm{GPa}$ for mature bone tissue $[23,57]$.

Table 3 : Sensitivity study on the averaged Young's modulus E (GPa) within newly formed (NF) and mature (M) bone and its relative variation $\Delta \mathrm{E} / \mathrm{E}_{0}$ between NF and $\mathrm{M}$ bone after 7 and 13 weeks of healing, for Poisson's ratio equal to $0.2,0.3$ and 0.4 .

\begin{tabular}{|c|c|c|c|c|c|c|}
\hline \multirow{2}{*}{$\begin{array}{c}\text { Poisson's } \\
\text { ratio }\end{array}$} & \multicolumn{3}{|c|}{7 weeks } & \multicolumn{3}{c|}{ 13 weeks } \\
\cline { 2 - 7 } & $\mathbf{N F}$ & $\mathbf{M}$ & $\Delta E / E_{0}$ & $\mathbf{N F}$ & $\mathbf{M}$ & $\Delta E / E_{0}$ \\
\hline $\mathbf{0 . 2}$ & 10.75 & 14.62 & 30.5 & 12.33 & 14.80 & 18.3 \\
\hline $\mathbf{0 . 3}$ & 10.19 & 13.86 & 30.5 & 11.68 & 14.03 & 18.3 \\
\hline $\mathbf{0 . 4}$ & 9.41 & 12.79 & 30.5 & 10.79 & 12.95 & 18.3 \\
\hline
\end{tabular}

The values of wave velocity $v$ are comprised between $4.77 \times 10^{3}$ and $5.41 \times 10^{3} \mathrm{~m} / \mathrm{s}$ and are in agreement with the previous study on the same in vivo model [16] and in the same range as the results obtained in other studies characterizing wave velocities within bone trabeculae $[41,42]$.

For most groups, the values of $E^{*}$ (Fig. 5) and $v$ (Fig. 6) are higher after 13 weeks than after 7 weeks of healing time, which is in agreement with the results obtained with a similar implant model [45]. Furthermore, the values of $E^{*}$ (Fig. 5A) and $v$ (Fig. 6A) increase between newly formed and mature cortical bone. Consequently, the increase of $E^{*}$ and $v$ with healing time and between newly formed and mature bone, as well as the constant values obtained in mature bone, lead to larger differences of $E, v$ and $\rho$ (see Table 2 ) after 7 weeks than after 13 weeks of healing time. For example, $\rho$ increases by $14.3 \%$ (respectively $6.1 \%$ ) between newly formed and mature bone tissues after 7 weeks of healing time (respectively 13 weeks of healing time), which is comparable to the increase of $12.2 \%$ (respectively $2.2 \%$ ) found in [45].

The gradual increase of $E, v$ and $\rho$ from 7 to 13 weeks of healing time and from newly formed to mature cortical bone tissue may be explained by the increase of mineralization occurring during 
osseointegration phenomena [5]. Previous nanoindentation studies have shown that the bone elastic modulus increases as a function of healing time using the same in vivo model [37], a titanium plate model [36], around dental implant [33] and within bone defects [23]. Furthermore, a recent study combining nanoindentation with Raman spectroscopy measurements showed that the increase of the bone elastic modulus is related to the increase of the degree of mineralization during osseointegration [58]. The aforementioned experimental results are in agreement with a homogenization model showing a non-linear increase of the Young's modulus as a function of bone tissue mineralization [59, 60]. Moreover, wave velocity also increases as a function of bone tissue mineralization, in line with results of previous micro-Brillouin scattering studies $[16,40,41,43]$.

The increase of bone mass density as a function of healing time and between newly formed and mature bone can be explained by an increased degree of mineralization, since bone mineral is denser than other bone components (collagen fibers and water) [37], consistently with bone tissue maturation [61]. Note that since the velocity $v$ can be modeled by a ratio between $E$ and $\rho$ (Eq. (3)), its relative variations are lower compared to $E$. Therefore, only variations of $E$ and $\rho$ will be considered in what follows.

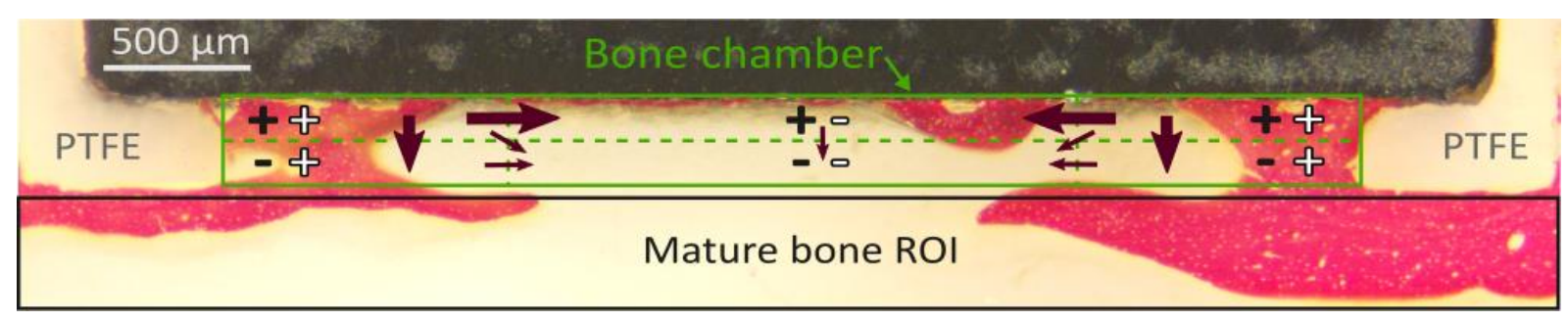

Figure 7 : Schematic representation of the spatial variation of $E$ and $\rho$ in each region of interest (ROI) of the bone chamber. The black (respectively white) symbols correspond to a comparison between the close and far (respectively lateral and central) groups. The ' + ' (respectively ' - ') sign indicates the ROI where E and $\rho$ are higher (respectively lower). The large arrows correspond to the direction of bone growth occurring initially and the small arrows represent later bone spreading.

Analyzing the spatial variations of $E$ and $\rho$ is a simple way to understand the course of osseointegration phenomena in the bone chamber. Since bone mineralization increases with healing time, the increase of $E$ and $\rho$ as a function of healing time (Fig. 5 and Table 2) can be considered as a marker of osseointegration phenomena. The schematic comparison of the values of $E$ and $\rho$ as a function of the position in the bone chamber shown in Fig. 7 emphasizes that bone tissue in the close group (black ' + ' signs) and in the lateral group (white ' + ' signs) is more mineralized and thus older compared to bone tissue located in the far group (black '-' signs) and in the central group (white '-' signs). Combining the 
close-to-far and the lateral-to-central evolutions of bone properties indicates that the highest bone properties are obtained in the upper corners of the bone chamber, which suggests that bone creation initiates in L1. The initial bone formation in L1 may be explained by the fact that L1 corresponds to locations where the local stress field is likely to be the most important compared to other ROIs since mechanical loading is known to trigger bone formation [2]. This result is in agreement with a previous paper [8] showing that the bone elastic properties measured using nanoindentation around a dental implant are higher around the upper parts of implant threading, where stresses are the most important.

After being created in L1, bone tissue then grows along the implant surface and towards mature bone tissue before spreading to the most central region of the bone chamber (arrows in Fig. 7). Such scenario is consistent with the variation of $B A / T A$ values shown in Fig. 4 indicating a larger amount of bone close to the implant and in the lateral region than in the far and central regions.

The aforementioned osseointegration scenario may be discussed in the light of the description of "contact" and "distance" osteogenesis described in the literature [62-65]. Contact osteogenesis corresponds to bone growth from the implant surface to the pre-existing bone tissue after several healing stages. Briefly, contact osteogenesis leads to the recruitment of osteogenic cells at the implant surface by osteoconduction, which will synthetize new bone through a woven collagenous matrix, later mineralized into bone. This whole process will spatially progress until bridging with the host bone [62, 65]. In parallel to contact osteogenesis, distance osteogenesis is a phenomenon corresponding to bone ingrowth from the pre-existing bone to the implant surface. In this case, the osteogenic cells are provided by the host bone which remodels following surgery $[62,65]$. Our results showing a bone formation starting in the upper corners of the bone chamber seem in better agreement with the contact osteogenesis scenario, since blood is likely to accumulate in L1 after surgery, providing the osteogenic cells needed for bone formation $[62,65]$. A predominant contact osteogenesis is expected when rougher surfaces are employed [66], as such surfaces offer more space for cells to attach to the implant surface, thus promoting bone cell migration [67]. Furthermore, bone does not only grow in contact with titanium, but also along PTFE on the lateral sides of the bone chamber. This second material may interfere with the spatial evolution of bone tissue and future studies should consider the effect of changing PTFE into another biomaterial.

This study has several limitations. First, the coin-shaped implant model leads to a relatively low level of mechanical loading, which limits osseointegration phenomena but is also representative of the clinical healing phase just after dental implant surgery. Moreover, the aim of this implant model is to create a bone chamber in order to obtain a clear distinction between mature and newly formed bone 
tissues and to work under standardized conditions. Second, the sample size remains relatively low because of the in vivo model and of time-consuming measurements. However, the present study has doubled the number of samples previously analyzed [45], which allows the evaluation of spatial variations. Third, nanoindentation and micro-Brillouin scattering analyses were performed on two consecutive slices because the thickness of the slices needed to be different. Fourth, the resin embedding using formalin and dehydrating the sample may alter bone nanostructure [68] and hence affect bone mechanical properties. In particular, the elastic stiffness is known to increase with bone dehydration [69]. However, it has been shown that comparative studies can be performed when all samples are embedded in a similar way because all samples or regions of interest are affected in a similar way [21]. The analyses carried out herein rely on relative variations among specimens. Furthermore, such procedure is essential in order to slice specimens containing metal without damaging the bone-implant interface, and represents a common procedure conducted in the literature [33, 35-37]. Fifth, although bone tissue is known to have an anisotropic mechanical behavior [29, 30], the isotropic assumption was made herein for the bone tissue constitutive law. Similar assumption was made for nanoindentation analyses in the literature $[8,19,31,35]$, which is particularly justified for newly formed bone because woven bone structure is disordered [70] and its mineral crystals are distributed isotropically $[71,72]$. The isotropic assumption is particularly important in order to derive the variation of mass density. However, nanoindentation and micro-Brillouin scattering are performed in two orthogonal directions. Here, we assumed similar relative variations of all components of the stiffness tensor. Future studies should consider the anisotropic behavior of the bone tissue.

\section{Conclusion}

This study combining nanoindentation, micro-Brillouin scattering, histology and a dedicated in vivo model allows the comparison of newly formed and mature cortical bone tissues and the evaluation of the spatio-temporal evolution of newly formed bone properties by considering different regions of interest within the bone chamber filled with newly formed bone. Bone properties are found to be higher close to the implant surface and in the lateral ROI than in the far and central ROls, as well as after longer healing times suggesting a higher degree of mineralization consistent with bone tissue maturation. These results lead to a bone spreading path confirmed by histology and consistent with contact osteogenesis. Identifying how bone develops around the BII provides a better understanding of osseointegration phenomena, which is likely to improve implant surgical outcomes. 


\section{Acknowledgements}

This work has received funding from the European Research Council (ERC) under the European Union's Horizon 2020 research and innovation program (grant agreement no 682001, project ERC Consolidator Grant 2015 Bonelmplant).

This project has received funding from the European Union's Horizon 2020 research and innovation programme under the Marie Sklodowska-Curie grant agreement No 797764.

\section{Conflicts of Interest}

The authors state that there is no conflict of interest.

\section{References}

[1] Huja, S. S., Katona, T. R., Burr, D. B., Garetto, L. P., and Roberts, W. E., 1999, "Microdamage adjacent to endosseous implants," Bone, 25(2), pp. 217-222.

[2] Haiat, G., Wang, H. L., and Brunski, J., 2014, "Effects of biomechanical properties of the boneimplant interface on dental implant stability: from in silico approaches to the patient's mouth," Annu Rev Biomed Eng, 16, pp. 187-213.

[3] Luo, G., Sadegh, A. M., Alexander, H., Jaffe, W., Scott, D., and Cowin, S. C., 1999, "The effect of surface roughness on the stress adaptation of trabecular architecture around a cylindrical implant," J Biomech, 32(3), pp. 275-284.

[4] Mathieu, V., Vayron, R., Richard, G., Lambert, G., Naili, S., Meningaud, J. P., and Haiat, G., 2014, "Biomechanical determinants of the stability of dental implants: influence of the bone-implant interface properties," J Biomech, 47(1), pp. 3-13.

[5] Gao, X., Fraulob, M., and Haiat, G., 2019, "Biomechanical behaviours of the bone-implant interface: a review," J R Soc Interface, 16(156), p. 20190259.

[6] Shah, F. A., Snis, A., Matic, A., Thomsen, P., and Palmquist, A., 2016, "3D printed Ti6Al4V implant surface promotes bone maturation and retains a higher density of less aged osteocytes at the bone-implant interface," Acta Biomater, 30, pp. 357-367.

[7] Tornquist, E., Isaksson, H., and Turunen, M. J., 2019, "Mineralization of cortical bone during maturation and growth in rabbits," J Bone Miner Metab. 
[8] Yoon, H. I., Jeon, M. J., Kim, H. L., Kim, D. G., and Han, J. S., 2018, "Spatial variation of bone biomechanical properties around a dental implant using nanoindentation: a case study," J Mech Behav Biomed Mater, 79, pp. 168-172.

[9] Chang, M. C., Ko, C. C., Liu, C. C., Douglas, W. H., DeLong, R., Seong, W. J., Hodges, J., and An, K. N., 2003, "Elasticity of alveolar bone near dental implant-bone interfaces after one month's healing," J Biomech, 36(8), pp. 1209-1214.

[10] Branemark, R., Ohrnell, L. O., Skalak, R., Carlsson, L., and Branemark, P. I., 1998, "Biomechanical characterization of osseointegration: an experimental in vivo investigation in the beagle dog," J Orthop Res, 16(1), pp. 61-69.

[11] Ronold, H. J., Lyngstadaas, S. P., and Ellingsen, J. E., 2003, "Analysing the optimal value for titanium implant roughness in bone attachment using a tensile test," Biomaterials, 24(25), pp. 45594564.

[12] Ronold, H. J., and Ellingsen, J. E., 2002, "Effect of micro-roughness produced by TiO2 blasting-tensile testing of bone attachment by using coin-shaped implants," Biomaterials, 23(21), pp. 42114219.

[13] Ronold, H. J., Lyngstadaas, S. P., and Ellingsen, J. E., 2003, "A study on the effect of dual blasting with $\mathrm{TiO} 2$ on titanium implant surfaces on functional attachment in bone," J Biomed Mater Res A, 67(2), pp. 524-530.

[14] Ronold, H. J., Ellingsen, J. E., and Lyngstadaas, S. P., 2003, "Tensile force testing of optimized coin-shaped titanium implant attachment kinetics in the rabbit tibiae," J Mater Sci Mater Med, 14(10), pp. 843-849.

[15] Ronold, H. J., and Ellingsen, J. E., 2002, "The use of a coin shaped implant for direct in situ measurement of attachment strength for osseointegrating biomaterial surfaces," Biomaterials, 23(10), pp. 2201-2209.

[16] Mathieu, V., Fukui, K., Matsukawa, M., Kawabe, M., Vayron, R., Soffer, E., Anagnostou, F., and Haiat, G., 2011, "Micro-Brillouin scattering measurements in mature and newly formed bone tissue surrounding an implant," J Biomech Eng, 133, p. 021006.

[17] Mathieu, V., Vayron, R., Soffer, E., Anagnostou, F., and Haiat, G., 2012, "Influence of healing time on the ultrasonic response of the bone-implant interface," Ultrasound Med Biol, 38(4), pp. 611618.

[18] Mathieu, V., Vayron, R., Barthel, E., Dalmas, D., Soffer, E., Anagnostou, F., and Haiat, G., 2012, "Mode III cleavage of a coin-shaped titanium implant in bone: effect of friction and crack propagation," J Mech Behav Biomed Mater, 8, pp. 194-203. 
[19] Willems, N. M., Mulder, L., den Toonder, J. M., Zentner, A., and Langenbach, G. E., 2014, "The correlation between mineralization degree and bone tissue stiffness in the porcine mandibular condyle," J Bone Miner Metab, 32(1), pp. 29-37.

[20] Pathak, S., Vachhani, S. J., Jepsen, K. J., Goldman, H. M., and Kalidindi, S. R., 2012, "Assessment of lamellar level properties in mouse bone utilizing a novel spherical nanoindentation data analysis method," J Mech Behav Biomed Mater, 13, pp. 102-117.

[21] Isaksson, H., Malkiewicz, M., Nowak, R., Helminen, H. J., and Jurvelin, J. S., 2010, "Rabbit cortical bone tissue increases its elastic stiffness but becomes less viscoelastic with age," Bone, 47(6), pp. $1030-1038$.

[22] Wolfram, U., Wilke, H. J., and Zysset, P. K., 2010, "Valid micro finite element models of vertebral trabecular bone can be obtained using tissue properties measured with nanoindentation under wet conditions," J Biomech, 43(9), pp. 1731-1737.

[23] Anesi, A., Ferretti, M., Cavani, F., Salvatori, R., Bianchi, M., Russo, A., Chiarini, L., and Palumbo, C., 2018, "Structural and ultrastructural analyses of bone regeneration in rabbit cranial osteotomy: Piezosurgery versus traditional osteotomes," J Craniomaxillofac Surg, 46(1), pp. 107-118.

[24] Mora-Macias, J., Pajares, A., Miranda, P., Dominguez, J., and Reina-Romo, E., 2017, "Mechanical characterization via nanoindentation of the woven bone developed during bone transport," J Mech Behav Biomed Mater, 74, pp. 236-244.

[25] Pelled, G., Tai, K., Sheyn, D., Zilberman, Y., Kumbar, S., Nair, L. S., Laurencin, C. T., Gazit, D., and Ortiz, C., 2007, "Structural and nanoindentation studies of stem cell-based tissue-engineered bone," J Biomech, 40(2), pp. 399-411.

[26] Tai, K., Pelled, G., Sheyn, D., Bershteyn, A., Han, L., Kallai, I., Zilberman, Y., Ortiz, C., and Gazit, D., 2008 , "Nanobiomechanics of repair bone regenerated by genetically modified mesenchymal stem cells," Tissue engineering. Part A, 14(10), pp. 1709-1720.

[27] Tai, K., Qi, H. J., and Ortiz, C., 2005, "Effect of mineral content on the nanoindentation properties and nanoscale deformation mechanisms of bovine tibial cortical bone," J Mater Sci Mater Med, 16(10), pp. 947-959.

[28] Tai, K., Ulm, F. J., and Ortiz, C., 2006, "Nanogranular origins of the strength of bone," Nano Lett, 6(11), pp. 2520-2525.

[29] Carnelli, D., Gastaldi, D., Sassi, V., Contro, R., Ortiz, C., and Vena, P., 2010, "A finite element model for direction-dependent mechanical response to nanoindentation of cortical bone allowing for anisotropic post-yield behavior of the tissue," J Biomech Eng, 132(8), p. 081008.

[30] Carnelli, D., Vena, P., Dao, M., Ortiz, C., and Contro, R., 2013, "Orientation and size-dependent mechanical modulation within individual secondary osteons in cortical bone tissue," J R Soc Interface, 10(81), p. 20120953. 
[31] Anchieta, R. B., Baldassarri, M., Guastaldi, F., Tovar, N., Janal, M. N., Gottlow, J., Dard, M., Jimbo, R., and Coelho, P. G., 2014, "Mechanical property assessment of bone healing around a titanium-zirconium alloy dental implant," Clin Implant Dent Relat Res, 16(6), pp. 913-919.

[32] Kim, D. G., Elias, K. L., Jeong, Y. H., Kwon, H. J., Clements, M., Brantley, W. A., Lee, D. J., and Han, J. S., 2016, "Differences between buccal and lingual bone quality and quantity of peri-implant regions," J Mech Behav Biomed Mater, 60, pp. 48-55.

[33] Johnson, T. B., Siderits, B., Nye, S., Jeong, Y. H., Han, S. H., Rhyu, I. C., Han, J. S., Deguchi, T., Beck, F. M., and Kim, D. G., 2018, "Effect of guided bone regeneration on bone quality surrounding dental implants," J Biomech, 80, pp. 166-170.

[34] Anchieta, R. B., Guimaraes, M. V. M., Suzuki, M., Tovar, N., Bonfante, E. A., Atria, P., and Coelho, P. G., 2018, "Nanomechanical Assessment of Bone Surrounding Implants Loaded for 3 Years in a Canine Experimental Model," J Oral Maxillofac Surg, 76(1), pp. 71-79.

[35] Kim, D. G., Kwon, H. J., Jeong, Y. H., Kosel, E., Lee, D. J., Han, J. S., Kim, H. L., and Kim, D. J., 2016, "Mechanical properties of bone tissues surrounding dental implant systems with different treatments and healing periods," Clin Oral Investig, 20(8), pp. 2211-2220.

[36] Claffey, N., Bashara, H., O'Reilly, P., and Polyzois, I., 2015, "Evaluation of New Bone Formation and Osseointegration Around Subperiosteal Titanium Implants with Histometry and Nanoindentation," Int J Oral Maxillofac Implants, 30(5), pp. 1004-1010.

[37] Vayron, R., Barthel, E., Mathieu, V., Soffer, E., Anagnostou, F., and Haiat, G., 2012, "Nanoindentation measurements of biomechanical properties in mature and newly formed bone tissue surrounding an implant," J Biomech Eng, 134(2), p. 021007.

[38] Matsukawa, M., Tsubota, R., Kawabe, M., and Fukui, K., 2014, "Application of a micro-Brillouin scattering technique to characterize bone in the GHz range," Ultrasonics, 54(5), pp. 1155-1161.

[39] Cardinalli, M. A., Dallari, D., Govoni, M., Stagni, F., Marmi, F., Tschon, M., Brogini, S., Fioretto, D., and Morresi, A., 2019, "Brillouin micro-spectroscopy of subchondral trabecular bone and articular cartilage of the human femoral head," Biomed Opt Express, 10(5), pp. 2606-2611.

[40] Akilbekova, D., Ogay, V., Yakupov, T., Sarsenova, M., Umbayev, B., Nurakhmetov, A., Tazhin, K., Yakovlev, V. V., and Utegulov, Z. N., 2018, "Brillouin spectroscopy and radiography for assessment of viscoelastic and regenerative properties of mammalian bones," J Biomed Opt, 23(9), pp. 1-11.

[41] Kawabe, M., Fukui, K., Matsukawa, M., Granke, M., Saied, A., Grimal, Q., and Laugier, P., 2012, "Comparative investigation of elastic properties in a trabecula using micro-Brillouin scattering and scanning acoustic microscopy," J Acoust Soc Am, 132(1), pp. EL54-60.

[42] Tsubota, R., Fukui, K., and Matsukawa, M., 2014, "Local ultrasonic wave velocities in trabeculae measured by micro-Brillouin scattering," J Acoust Soc Am, 135(2), pp. EL109-114. 
[43] Fukui, K., Takayanagi, S., Suga, D., and Matsukawa, M., 2012, "Measurement of wave velocity in cortical bone by micro-Brillouin scattering technique: Effect of bone tissue properties," Jpn J Appl Phys, 51(07GF20), pp. 1-2.

[44] Imoto, Y., Tsubota, R., Kawabe, M., Saito, M., Marumo, K., and Matsukawa, M., 2015, "Effects of abnormal collagen crosslinks on hypersonic longitudinal wave velocity in bovine cortical bone," Glycative Stress Research, 2(3), pp. 101-107.

[45] Vayron, R., Matsukawa, M., Tsubota, R., Mathieu, V., Barthel, E., and Haiat, G., 2014, "Evolution of bone biomechanical properties at the micrometer scale around titanium implant as a function of healing time," Phys Med Biol, 59(6), pp. 1389-1406.

[46] Palmquist, A., 2018, "A multiscale analytical approach to evaluate osseointegration," J Mater Sci Mater Med, 29(5), p. 60.

[47] Le Cann, S., Tudisco, E., Perdikouri, C., Belfrage, O., Kaestner, A., Hall, S., Tagil, M., and Isaksson, H., 2017, "Characterization of the bone-metal implant interface by Digital Volume Correlation of insitu loading using neutron tomography," J Mech Behav Biomed Mater, 75, pp. 271-278.

[48] Winter, W., Heckmann, S. M., and Weber, H. P., 2004, "A time-dependent healing function for immediate loaded implants," J Biomech, 37(12), pp. 1861-1867.

[49] Chevallier, N., Anagnostou, F., Zilber, S., Bodivit, G., Maurin, S., Barrault, A., Bierling, P., Hernigou, P., Layrolle, P., and Rouard, H., 2010, "Osteoblastic differentiation of human mesenchymal stem cells with platelet lysate," Biomaterials, 31(2), pp. 270-278.

[50] Soffer, E., Ouhayoun, J. P., Meunier, A., and Anagnostou, F., 2006, "Effects of autologous platelet lysates on ceramic particle resorption and new bone formation in critical size defects: the role of anatomical sites," J Biomed Mater Res B Appl Biomater, 79(1), pp. 86-94.

[51] Schneider, C. A., Rasband, W. S., and Eliceiri, K. W., 2012, "NIH Image to ImageJ: 25 years of image analysis," Nat Methods, 9(7), pp. 671-675.

[52] Doube, M., Klosowski, M. M., Arganda-Carreras, I., Cordelieres, F. P., Dougherty, R. P., Jackson, J. S., Schmid, B., Hutchinson, J. R., and Shefelbine, S. J., 2010, "BoneJ: Free and extensible bone image analysis in ImageJ," Bone, 47(6), pp. 1076-1079.

[53] Oliver, W. C., and Pharr, G. M., 2004, "Measurement of hardness and elastic modulus by instrumented indentation: Advances in understanding and refinements to methodology," J Mater Res, 19(1), pp. 3-20.

[54] Kruger, J. K., Embs, J., Brierley, J., and Jimenez, R., 1998, "A new Brillouin scattering technique for the investigation of acoustic and opto-acoustic properties: application to polymers," J Phys D: Appl Phys, 31, pp. 1913-1917. 
[55] Kawabe, M., Matsukawa, M., and Ohtori, N., 2010, "Measurement of wave velocity distribution in a trabecula by micro-Brillouin scattering technique," Jpn J Appl Phys, 49(07HB05), pp. 07HB05 0104.

[56] Zysset, P. K., Guo, X. E., Hoffler, C. E., Moore, K. E., and Goldstein, S. A., 1999, "Elastic modulus and hardness of cortical and trabecular bone lamellae measured by nanoindentation in the human femur," J Biomech, 32(10), pp. 1005-1012.

[57] Bianchi, M., Boi, M., Sartori, M., Giavaresi, G., Lopomo, N., Fini, M., Dediu, A., Tampieri, A., Marcacci, M., and Russo, A., 2015, "Nanomechanical mapping of bone tissue regenerated by magnetic scaffolds," J Mater Sci Mater Med, 26(1), p. 5363.

[58] Fraulob, M., Pang, S., Le Cann, S., Vayron, R., Laurent-Brocq, M., Todatry, S., Soares, J. A. N. T., Jasiuk, I., and Haiat, G., submitted, "Multimodal characterization of the bone-implant interface using Raman spectroscopy and nanoindentation," Med Eng Phys.

[59] Sansalone, V., Bousson, V., Naili, S., Bergot, C., Peyrin, F., Laredo, J. D., and Haiat, G., 2012, "Anatomical distribution of the degree of mineralization of bone tissue in human femoral neck: impact on biomechanical properties," Bone, 50(4), pp. 876-884.

[60] Sansalone, V., Naili, S., Bousson, V., Bergot, C., Peyrin, F., Zarka, J., Laredo, J. D., and Haiat, G., 2010, "Determination of the heterogeneous anisotropic elastic properties of human femoral bone: from nanoscopic to organ scale," J Biomech, 43(10), pp. 1857-1863.

[61] Isaksson, H., Harjula, T., Koistinen, A., livarinen, J., Seppanen, K., Arokoski, J. P., Brama, P. A., Jurvelin, J. S., and Helminen, H. J., 2010, "Collagen and mineral deposition in rabbit cortical bone during maturation and growth: effects on tissue properties," J Orthop Res, 28(12), pp. 1626-1633.

[62] Kuzyk, P. R., and Schemitsch, E. H., 2011, "The basic science of peri-implant bone healing," Indian journal of orthopaedics, 45(2), pp. 108-115.

[63] Puleo, D. A., and Nanci, A., 1999, "Understanding and controlling the bone-implant interface," Biomaterials, 20(23-24), pp. 2311-2321.

[64] Berglundh, T., Abrahamsson, I., Lang, N. P., and Lindhe, J., 2003, "De novo alveolar bone formation adjacent to endosseous implants," Clin Oral Implants Res, 14(3), pp. 251-262.

[65] Davies, J. E., 2003, "Understanding peri-implant endosseous healing," Journal of dental education, 67(8), pp. 932-949.

[66] Choi, J. Y., Albrektsson, T., Jeon, Y. J., and Yeo, I. L., 2019, "Osteogenic Cell Behavior on Titanium Surfaces in Hard Tissue," Journal of clinical medicine, 8(5), pp. 1-10.

[67] Han, G., and Shen, Z., 2015, "Microscopic view of osseointegration and functional mechanisms of implant surfaces," Mater Sci Eng C Mater Biol Appl, 56, pp. 380-385. 
[68] Fiedler, I. A. K., Casanova, M., Keplinger, T., Busse, B., and Muller, R., 2018, "Effect of shortterm formaldehyde fixation on Raman spectral parameters of bone quality," J Biomed Opt, 23(11), p. 116504.

[69] Hengsberger, S., Kulik, A., and Zysset, P., 2002, "Nanoindentation discriminates the elastic properties of individual human bone lamellae under dry and physiological conditions," Bone, 30(1), pp. 178-184.

[70] Shah, F. A., Thomsen, P., and Palmquist, A., 2019, "Osseointegration and current interpretations of the bone-implant interface," Acta Biomater, 84, pp. 1-15.

[71] Tsai, P.-I., Lam, T.-N., Wu, M.-H., Tseng, K.-Y., Chang, Y.-W., Sun, J.-S., Li, Y.-Y., Lee, M.-H., Chen, S.-Y., Chang, C.-K., Su, C.-J., Lin, C.-H., Chiang, C.-Y., Ku, C.-S., Tsou, N.-T., Shih, S.-J., Wang, C.-C., and Huang, E.-W., 2019, "Multi-scale mapping for collagen-regulated mineralization in bone remodeling of additive manufacturing porous implants," Mater Chem Phys, 230, pp. 83-92.

[72] Takano, Y., Turner, C. H., and Burr, D. B., 1996, "Mineral anisotropy in mineralized tissues is similar among species and mineral growth occurs independently of collagen orientation in rats: results from acoustic velocity measurements," J Bone Miner Res, 11(9), pp. 1292-1301. 